\title{
Evaluación del efecto del biol sobre catorce accesiones de papa nativa (Solanum ssp.) en la estación experimental kallutaca
}

\author{
Evaluation of biol's effect on fourteen accession of native potato (Solanum ssp.) at the \\ experimental station kallutaca
}

Condori-Mamani Pastor ${ }^{1,2 *}$, Loza-Murguia Manuel Gregorio ${ }^{3,4}$, Sainz-Mendoza Humberto Nelson ${ }^{1,2}$, Guzmán-Calla Jorge $^{1,2}$, Mamani-Pati Francisco ${ }^{1,2}$, Marza-Mamani Félix ${ }^{1,2}$, Gutiérrez-Gonzáles Diego Eddy ${ }^{1,2}$

\begin{tabular}{l} 
Datos del Articulo \\
\hline 1'Universidad Pública de El Alto-UPEA, \\
Área de Ciencias Agrícolas, Pecuarias y \\
Recursos Naturales, Ingeniería Agronómi- \\
ca. Laja-Los Andes. La Paz, Estado \\
Plurinacional de Bolivia. (591)2-2115231. \\
2 Instituto de Investigación y Extensión \\
Agrícola (IINEA). Área de Ciencias \\
Agrícolas, Pecuarias y Recursos Natura- \\
les, Ingeniería Agronómica. \\
Universidad Católica Boliviana San \\
Pablo-UCB, Unidad Académica Campe- \\
sina Carmen Pampa-UAC-CP, Ingeniería \\
Agronómica. Coroico. Nor Yungas. La \\
Paz, Estado Plurinacional de Bolivia. \\
591(2)8781991. \\
${ }^{4}$ Departamento de Enseñanza e Investiga- \\
ción en Bioquímica \& Microbiología- \\
DEI\&BM. Unidad Académica Campesina \\
Carmen Pampa-UAC-CP. \\
boliviamanloza@ yahoo.com \\
*Dirección de contacto: \\
Pastor Condori-Mamani. \\
Instituto de Investigación y Extensión \\
Agrícola, Inge-niería Agronómica, \\
Universidad Pública de El Alto (UPEA). \\
Km 25, Carretera Internacional La Paz- \\
Desaguadero. \\
La Paz - Estado Plurinacional de Bolivia. \\
Tel +591-73515847. \\
E-mail: pmcondori@gmail.com \\
Palabras clave: \\
Accesiones, \\
biol, \\
papa nativa, \\
fito-estimulante, \\
rendimiento. \\
\hline
\end{tabular}

J Selva Andina Biosph. 2017; 5(1):15-28.

\section{Historial del artículo.}

Recibido enero, 2014

Devuelto septiembre 2014

Aceptado septiembre, 2016

Disponible en línea, mayo 2017.

Editado por:

Selva Andina

Research Society
Resumen

En Bolivia las regiones productoras de papa, se caracterizas por una agricultura extensiva en mantas (de forma tradicional), de auto subsistencia y poca diversificada, la mayor parte de los productores poseen pequeñas propiedades para el cultivo de papa, e ubican en pequeñas parcelas que anualmente van rotando.

El presente trabajo de investigación tiene el objetivo de comparar y caracterizar catorce accesiones de papa nativa de la colección de germoplasma, de la Carrera de Ingeniería Agronómica, (UPEA). El estudio se llevó acabo en la estación experimental de Kallutaca, estableciéndose en base a un Diseño Bloques Completos al Azar (DBCA) con tres repeticiones cada accesión, el segundo factor es la dosis de biol aplicado, se consideró como un fito-estimulante complejo, para los cultivos, donde permiten mayor incrementos en la capacidad de fotosíntesis y la producción. Cuyos valores se sitúan entre 2.0 a $2.6 \%$ (\% en material seco) y que reportaron una concentración de $\mathrm{N}$ total de 3.3 a $3.7 \%$. El análisis de micro nutriente en el efluente obtenido detectó la presencia de Hierro (132 ppm), Manganeso, Cobre y Zinc con 12.1 y 6 ppm. Las accesiones "Polo", "Sacampaya", "Sani imilla", "Janq’o choque", "Bola runa", "Q'aysalla” y "Laramq'aysa" tuvieron mayor desarrollo, la misma tienen mayores rendimientos los restantes no tuvieron la misma incidencia.

Esta respuesta se debe a un efecto positivo del biol sobre el rendimiento de tubérculos conforme a dosis de biol aplicado a las accesiones de papa nativa donde se obtuvo un rendimiento equivalente a 10 t.ha $^{-1}$ con la aplicación de dosis de $25 \%$ de Biol.

(C) 2017. Journal of the Selva Andina Biosph. Bolivia. Todos los derechos reservados.

\section{Abstract}

In Bolivia the productive potato regions, you characterize for an extensive agriculture in blankets (of traditional form), of car subsistence and not much diversified, the little properties for the cultivation of potato possess the greater part of the productive, and they locate at little plots of land that annually they go rotating.

The present research work has the objective to make a comparison and to characterize fourteen accessions of native potato of germoplasma's collection, of Agronomic Ingeniería's race, (UPEA). The study carried completion at Kallutaca's experiment station itself, becoming established on the basis of a Design Bloques Complete at random (DBCA) with three repetitions each accession, the second factor is diligent biol's dose, considered himself like a stimulant compound fito, for cultivations, where increments in the capability of photosynthesis and the production allow principal. Whose moral values place between $2.02 .6 \%$ themselves (\% in dry material) and that they yielded $3.3 \%$ 's concentration of total $\mathrm{N}$ to 
3.7\%. The analysis of microcomputer Hierro's (132 ppm), Manganeso's, Cobre's and Zinc's presence with 12.1 and 6 ppm detected nutrient in the obtained effluent.

Key words:

Accessions,

biol,

native potato,

stimulant fito,

performance.
Accessions Pole, Sacampaya, Sani imilla, Janq'o, collide, Runic Bola, Q'aysalla and Laramq'aysa had bigger development, the same the remainders have bigger performances they did not have the same incidence.

This answer is due to a positive effect of the biol on the performance of tubers in accordance with dose of diligent biol the accessions of native potato where ${ }^{1}$ with the application of dose of $25 \%$ of Biol obtained to 10 t.h $^{-1}$ an equivalent yield.

(C) 2017. Journal of the Selva Andina Biosph. Bolivia. All rights reserved.

\section{Introducción}

El género Solanum tiene más de 300 especies que forman tubérculos, entre especies cultivadas y silvestres, sin embargo el género, considerado altamente polimórfico y muy complejo (Ochoa 1990) incluye alrededor de 2400 especies alrededor del mundo (Ochoa 1999).

En Bolivia, la papa es típicamente cultivada en pequeñas superficies en las zonas altas e interandinas, en una superficie aproximada de 113375 ha, representando la principal fuente de ingresos de 125000 personas (Thiele et al. 2008). A pesar de la gran importancia que tiene en el país, la producción y productividad es baja, con un rendimiento promedio de 5.98 tha-1 (Zeballos et al. 2009), aunque en los últimos años, han mejorado significativamente los rendimientos en algunas zonas de altura y de valles en Cochabamba y Santa Cruz. Entre las razones que explican esta situación, se encuentran la falta de agua de riego, suelos pobres en materia orgánica, cultivares con bajo potencial de rendimiento, semilla de baja calidad, cultivo en zonas montañosas que dificultan la mecanización, además se tiene la presencia de plagas, enfermedades, heladas, granizo y sequía (Gabriel et al. 2011).

En los andes de Bolivia, los agricultores conservan muchos cultivares nativos de las ocho especies conocidas, las mismas que son usadas para su alimentación y seguridad alimentaria. Sin embargo, poco se conoce acerca de los atributos de resistencia a los factores bióticos y abióticos que afectan a la papa y menos aún se han utilizado en los programas de mejora genética de papa en el mundo (Estrada 2000, Gabriel 2010).

Está clara la importancia de la materia orgánica (MO) en las propiedades físicas y químicas del suelo, propiedades influidas por MO, incluyen, la estructura del suelo, capacidad de retención del agua, diversidad y actividad de organismos (tanto beneficioso como dañino para la producción) y disponibilidad de nutrientes. El incremento de $\mathrm{MO}$ en el suelo contribuye indirectamente a la porosidad del suelo (a través del incremento de la actividad de la fauna del suelo), de ahí el agua se infiltra rápidamente pudiendo ser retenida en el suelo (Bot \& Benites 2005).

El estiércol puede incrementar el contenido de MO, $\mathrm{pH}$, capacidad de intercambio catiónico y el nivel de nutrientes de la planta, incrementando la capacidad de retención de humedad del suelo, conductividad hidráulica, tamaño y cantidad de agregados hídro estables (Warren \& Fonteno 1994, Rojas-Rodriguez \& Ortuño 2007). Además, el humus de lombriz posee una mayor capacidad de retención de humedad que un suelo sin materia orgánica y mayor población de microorganismos que el compost (Gajalakshmi \& Abbasi 2004, Rojas-Rodriguez \& Ortuño 2007).

Un biofertilizante es una sustancia que contiene organismos vivos, que aplicados a la semilla, planta 
o suelo, colonizan la rizósfera y promueven su crecimiento a través del incremento de la provisión o disponibilidad de nutrientes primarios a la planta huésped. Así, las micorrizas como el Glomus fasciculatum facilitan la absorción de agua del suelo (Ortuño et al. 2010, Avilés et al. 2011), las bacterias como Bacillus spp., son promotores de crecimiento de la planta, que además las protegen de patógenos, permitiendo establecer una simbiosis para que las plantas sean vigorosas con mayor rendimiento.

En función a lo mencionado, la presente investigación ha sido orientada a la experimentación del Biol como fuente de fertilización orgánica de bajo costo, para el cultivo de papa, en los suelos del Altiplano boliviano, de ahí que el objetivo fue, determinar el efecto de la aplicación del Biol como fertilizante foliar en catorce accesiones de papas nativas (Solanum ssp.), en la Estación Experimental de Kallutaca.

\section{Materiales y métodos}

Localización. El presente trabajo se realizó en el año agrícola 2007-2008 en las instalaciones de la Estación Experimental de Kallutaca, dependiente de la Universidad Pública y Autónoma de El Alto (UPEA), ubicada en la provincia Los Andes, segunda sección municipal de Laja del departamento de La Paz, a 20 km de la Sede de Gobierno (Figura, 1). Geográficamente se encuentra situada a longitud Oeste $16^{\circ} 26^{\prime} 19.00^{\prime \prime}$ y $16^{\circ} 46^{\prime} 15.11^{\prime \prime}$ latitud Sur: $68^{\circ} 19^{\prime} 49.18^{\prime \prime}$ y $68^{\circ} 29^{\prime} 49.81$ y 3800 msnm.

Material vegetal. Catorce accesiones de papa nativa (Solanum tuberosum ssp.), procedentes de la colección de germoplasma de papa nativa de la Carrera de Ingeniería Agronómica del Universidad Pública y Autónoma de El Alto, (UPEA), corresponden a los códigos: (39, 74, 62, 76, 46, 77, 69, 71, 43, 92, 17
$85,80,47$ y 50), elegidas por su valor comercial, adaptación, rusticidad y productividad en las condiciones locales.

\section{Figura 1 Área de la ubicación de zona de estudio de estación experimental de kallutaca}

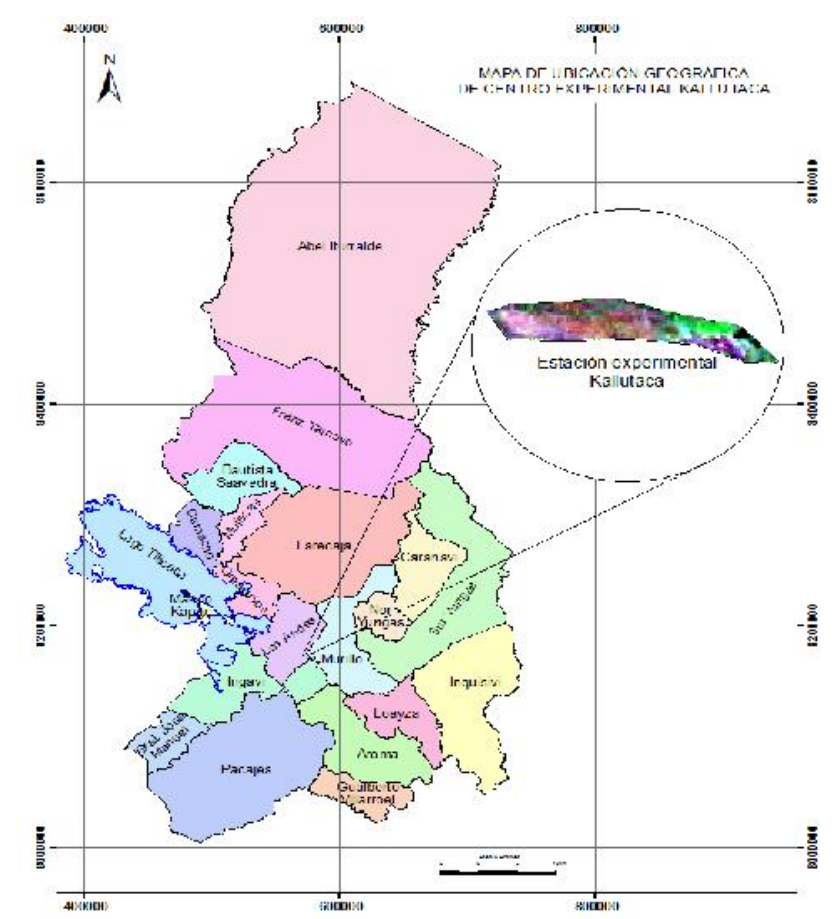

Preparación del biol. El material orgánico para la preparación del Biol, su composición (tabla 1).

Los materiales se mezclaron en tres biodigestores tipo turril de cierre hermético para mantener las condiciones anaeróbicas (figura 2), la alfalfa con el fin de balancear el contenido de nitrógeno, la cáscara de huevo, fue molida finamente para enriquecer con Calcio y Fósforo, se adicionó agua y se procedió a homogenizar. Los biodigestores fueron instalados bajo un ambiente protegido (carpa solar de agrofilm) para favorecer el proceso fermentativo. El metano fue purgado a través de una manguera, por burbujeo en una botella (figura 2) 
Tabla 1 El material orgánico para la preparación del Biol, su composición

\begin{tabular}{lc}
\hline \multicolumn{1}{c}{ Material } & Cantidad \\
\hline Agua & $125 \mathrm{~L}$ \\
Estiércol fresco & $50 \mathrm{Kg}$ \\
Gallinaza & $3 \mathrm{Kg}$ \\
Alfalfa (Medicao sativa) & $2 \mathrm{Kg}$ \\
Purín bovino & $2 \mathrm{~L}$ \\
Cascara de huevo & 10 Unidades \\
\hline
\end{tabular}

Obtención del efluente de la biodigestión. Después de 90 días de biodigestión, bajo las condiciones descritas. Por decantación y posterior filtrado, se extrajo el sobrenadante que fue enviado para su análisis fisicoquímico en el laboratorio del Centro de Investigación Agrícola Tropical (CIAT), dependiente de la Universidad Autónoma Gabriel Rene Moreno (UAGRM).

Figura 2 Ilustración de la preparación del Biol
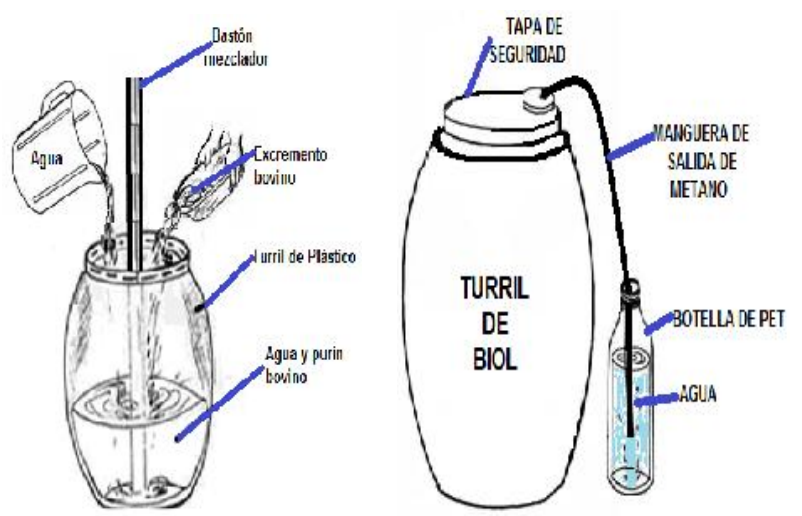

Fase de campo. Preparación del terreno: Se realizó con tractor agrícola en mayo del 2007, arando a una profundidad de 25 a $30 \mathrm{~cm}$. Posteriormente se realizó el mullido en el mes de septiembre de 2007 con la misma maquinaria. Siete días antes de la siembra se realizó una limpieza de malezas y piedras con posterior rastrillado y nivelado del terreno.
Delimitación del terreno: Los límites de los bloques y los tratamientos fueron delimitados con estacas y cordeles.

Siembra: Se realizó en fecha 28 de octubre de 2007. Para ello se procedió a la apertura de surcos a una profundidad de $20 \mathrm{~cm}$ aproximadamente, con distanciamiento de $80 \mathrm{~cm}$ entre surcos. Los tubérculos - semilla, se colocaron a una distancia de $25 \mathrm{~cm}$, por lo que en cada surco se distribuyeron 10 tubérculos haciendo un total de tres surcos por accesión de papa nativa. La siembra se realizó de forma manual, cubriendo posteriormente con suelo.

Aporque: A los 20 días de la emergencia, simultáneamente con el desmalezado, se efectuó el primer aporque, retirando suelo del entre surco y acercándolo a la línea de siembra con la finalidad de proporcionar a las plantas un mejor anclaje y evitar una excesiva humedad al pie de las mismas. Una vez que las plantas alcanzaron los $20 \mathrm{~cm}$ de altura, se procedió al segundo aporque de manera conjunta con la adición del fertilizante foliar, de un litro por metro cuadrados cada 20 días.

Aplicación de Biol: La aplicación foliar del producto obtenido (Biol) se realizó por aspersión (mochila aspersores manual) distribuyendo las distintas diluciones planteadas para los tratamientos del producto en el follaje del cultivo; Las aplicaciones se realizaron a partir de los 20 días de la emergencia; a intervalos sucesivos de 20 días hasta la cosecha, los cuales se aplicaron seis aplicaciones durante la fase vegetativa, la porción de 1 litro por seis $\mathrm{m}^{2}$ según la disolución plateado.

Cosecha: Se realizó cuando las hojas de la plantas se tornaron de color amarillento, a la vez que los tubérculos se desprendían con facilidad de sus estolones, indicadores de la madurez fisiológica del cultivo. Asimismo se tomó en cuenta el surgimiento de una piel más gruesa en el tubérculo cuyo propó- 
sito es brindar una mayor resistencia a patógenos que atacan durante el almacenamiento.

Clasificación y almacenamiento de tubérculos: Concluida la cosecha se efectuó una selección rigurosa de los tubérculos, separando aquellos afectados por cortes, heridas, síntomas y presencia de plagas, enfermedades $\mathrm{u}$ otras características atípicas. La selección de los tubérculos se realizó por tamaños y longitud del tubérculo de acuerdo a las siguientes categorías: Clase I $>5 \mathrm{~cm}$, Clase II de 4 a $5 \mathrm{~cm}$, Clase III de 3 a $4 \mathrm{~cm}$, Clase IV de 1.5 a $3 \mathrm{~cm}$ y finalmente Clase $\mathrm{V}<1.5 \mathrm{~cm}$.
Diseño experimental. Se adoptó un Diseño de Bloques Completos al Azar (DBCA), las accesiones fueron distribuidas aleatoriamente en las unidades experimentales, así como el factor "Biol" (diluciones). Las concentraciones de Biol aplicadas en cada bloque se muestran en tabla 2.

Tabla 2 Concentraciones de Biol utilizadas para la aplicación en trabajo de investigación

\begin{tabular}{lc}
\hline Tratamientos & Relación Agua: Biol \\
\hline T1 Agua: Biol & $(1 \%: 1 \%)$ \\
T2 Agua (Testigo) & - \\
T3 Agua: Biol & $(1 \%: 3 \%)$ \\
\hline
\end{tabular}

Figura 3 Croquis de distribución de los tratamientos

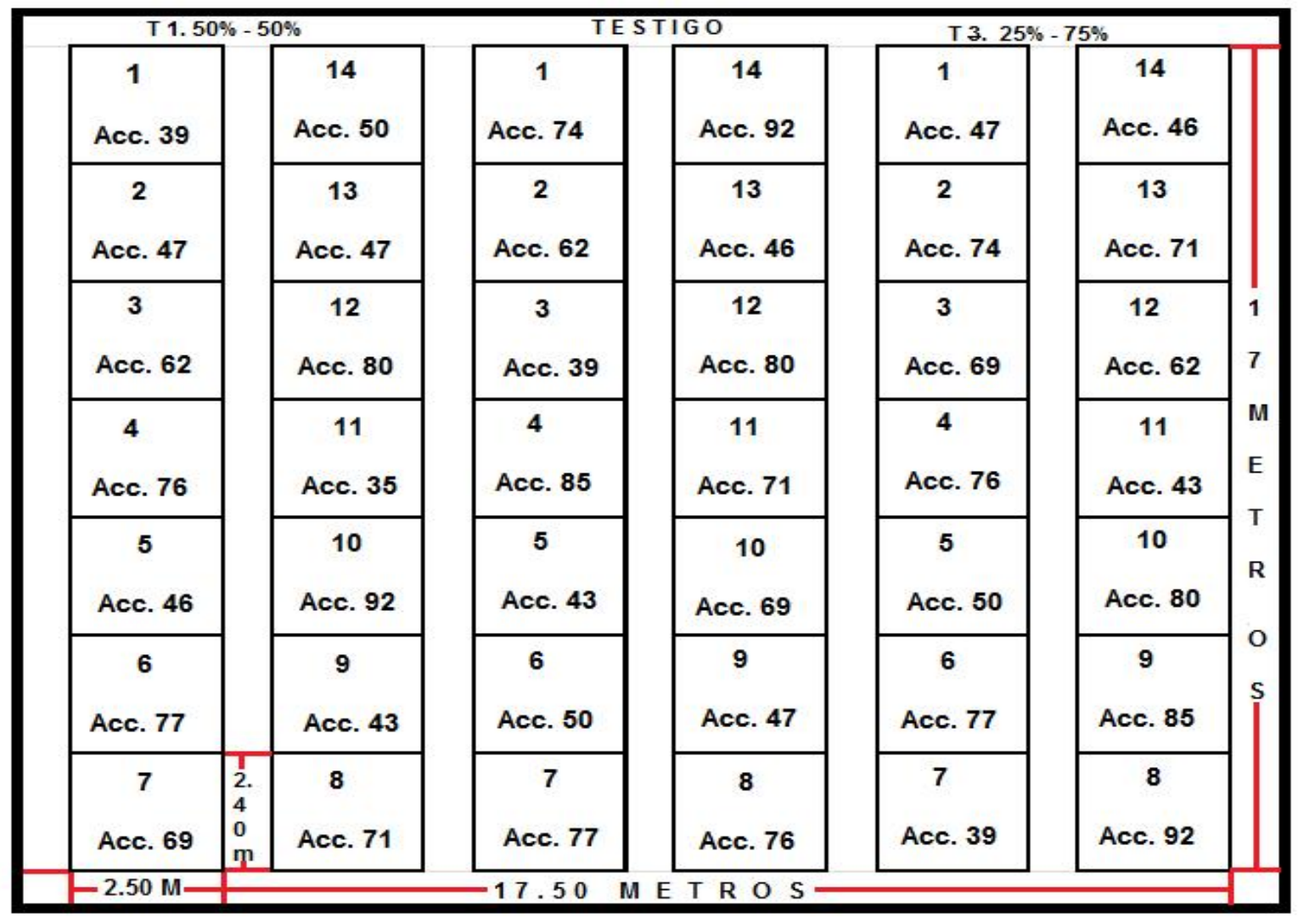

Las accesiones de papa fueron distribuidas al interior de cada bloque de acuerdo a la siguiente codificación. 39 Polo, Accesión 74 Canatilla, Accesión 62 Saq'anpaya, Accesión 76 Amajaya, Accesión 46 19
Alq’a pali (Leke Pek'e), Accesión 77 Sani imilla, Accesión 69 Janq`o choque pitu, Accesión 71 Imilla blanca (Janqo Imilla, Yuraj Imilla), Accesión 43 Polônia, Accesión 92 Bola runa, Accesión 85 Q'ay- 
salla, Accesión 80 Laram q'aysalla, Accesión 47 Chunculita (Surimana), Accesión 50 Ajahuiri.

El área total cultivable del ensayo fue de $297.5 \mathrm{~m}^{2}$. Las dimensiones para cada unidad experimental fueron: Ancho interno $2.50 \mathrm{~m}$, largo interno de parcela, $2.40 \mathrm{~m}$, área cultivable, por parcela $6 \mathrm{~m}^{2}$

\section{Modelo lineal}

Se aplicó el siguiente modelo lineal (Fisher et al. 1989)

$$
\mathrm{Y}_{\mathrm{ij}}=\mu+\beta_{\mathrm{j}}+\alpha_{\mathrm{i}}+\varepsilon_{\mathrm{ij}}
$$

Para:

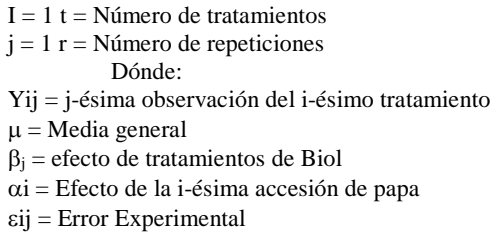

\section{Análisis estadístico}

Con los datos obtenidos se procedió al análisis de varianza (ANOVA) con un nivel de significancia $\alpha$ $=0.05 \%$. Para la comparación entre medias se utilizó la prueba de Duncan (5\%).

\section{Variables de respuesta}

Variables de respuesta en función a la aplicación de Biol

La respuesta frente a la aplicación de Biol se midió en términos de rendimiento en el cultivo de papa nativa.

\section{Variables fenológicas}

La evaluación de variables fenológicas se realizó durante un periodo de 180 días, de acuerdo a los periodos propuestos por (Huamán \& Spooner 2002), que establece un periodo de 160 a 175 días (para papas dulces) y de 170 a 180 días para papas amargas.

Días a la emergencia: Se determinaron los días a la emergencia (brotamiento), el mismo que ocurre entre 15 a 20 días después de la siembra.

Días a floración: Se consideró los días a la flora- ción, es decir cuando la corola de la flor se abre completamente, lo que generalmente ocurre de 20 a 25 días después de la emergencia.

Días de madurez fisiológica: Se determinaron los días transcurridos hasta que el cultivo alcanzó la madurez fisiológica, lo que ocurre generalmente entre 150 a 180 días después de la emergencia.

Variables Agronómicas. Altura de la planta (AP), largo de tallo (LT), número de ramas (NR), número de flores (NF), cobertura foliar (CF)

Variables de rendimiento. Peso del tubérculo (PT), Número de tubérculos por planta (NTP)

\section{Resultados}

\section{Caracterización fisicoquímica del biol}

Figura 4 composición química del "biol” en base a materia seca

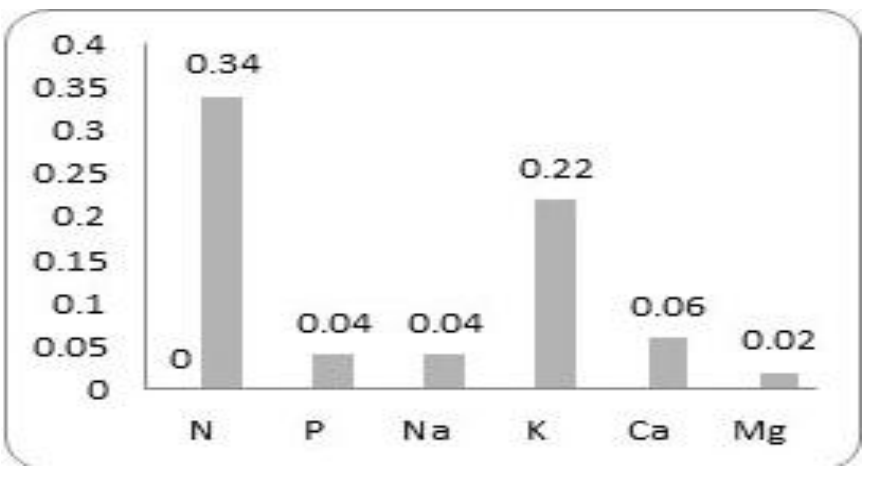

Figura 5 Concentración de micronutrientes en el biol

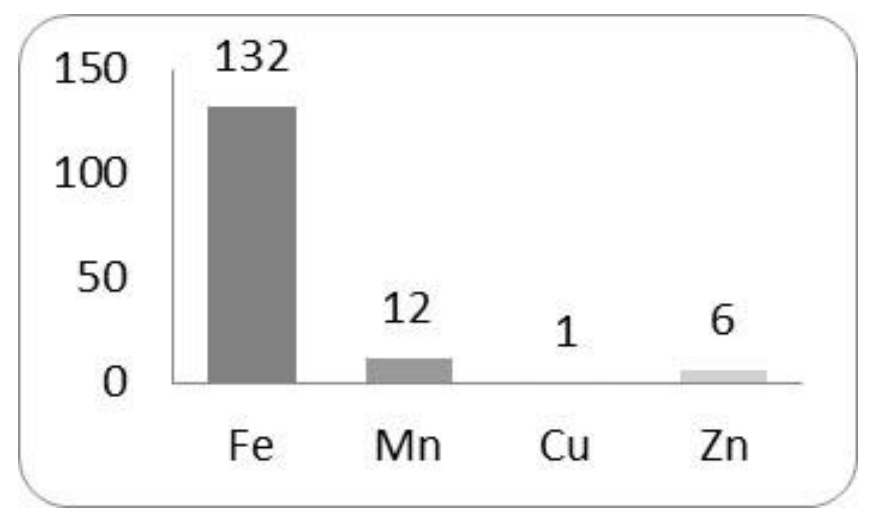


Tabla 3 Análisis de varianza para rendimientos e eficiencia de los tratamientos en la aplicación de biol en variables

\begin{tabular}{|c|c|c|c|c|c|c|c|c|c|}
\hline \multirow{2}{*}{$\begin{array}{c}\text { Fuente } \\
\text { variación }\end{array}$} & \multirow[t]{2}{*}{ GL } & \multicolumn{2}{|c|}{$\mathbf{A P}$} & \multicolumn{2}{|c|}{ LR } & \multicolumn{2}{|c|}{ NR } & \multicolumn{2}{|c|}{ NF } \\
\hline & & CM & Pr $>$ F & CM & $\operatorname{Pr}>\mathbf{F}$ & CM & $\operatorname{Pr}>\mathbf{F}$ & CM & $\operatorname{Pr}>\mathbf{F}$ \\
\hline BIOL (Bloq.) & 2 & 37.007 & 0.329 & 45.98 & 0.175 & 10.024 & 0.062 & 3.31 & 0.133 \\
\hline ACC & 13 & 101.74 & 0.006 & 60.714 & 0.025 & 11.817 & 0.002 & 3.663 & 0.027 \\
\hline Error & 26 & 24.692 & & 24.692 & & 3.229 & & 1.515 & \\
\hline Total & 41 & & & & & & & & \\
\hline $\mathrm{CV}$ & & & 19.34 & & 15.50 & & 20.51 & & 10.33 \\
\hline $\mathrm{R}^{2}$ & & & 0.628 & & 0.579 & & 0.674 & & 0.579 \\
\hline
\end{tabular}

Figura 6 Análisis de comparación de medias de Duncan $(\alpha=0.05)$ de catorce accesiones de papa nativa en estación experimental de kallutaca

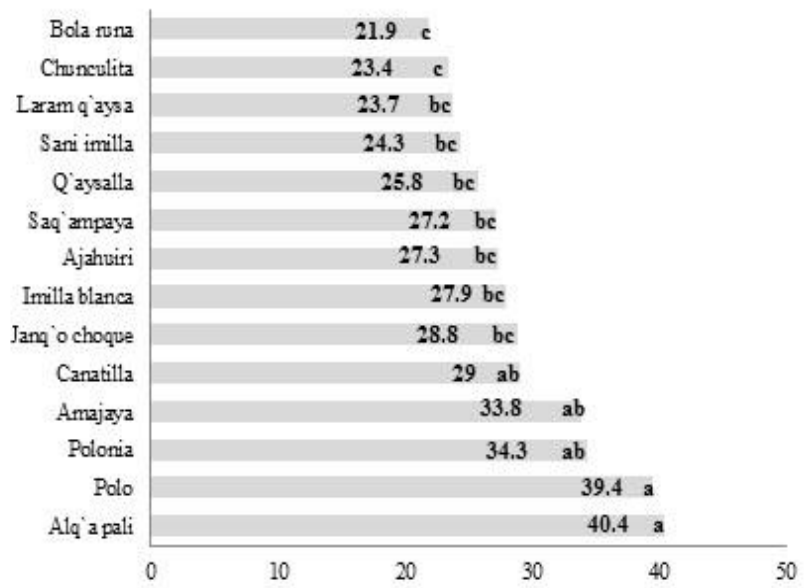

Figura 7 análisis de medias mediante la prueba de Duncan $(\alpha=0.05)$ largo de ramas para diferentes accesiones de papa nativa

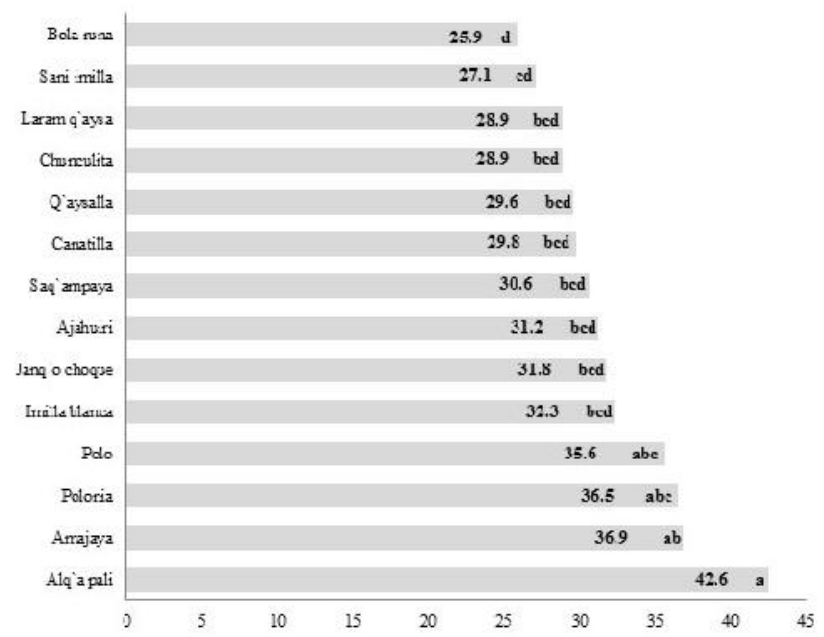

Figura 8 Aanálisis de comparación mediante la prueba de Duncan $(\alpha=0.05)$ número de ramas para diferentes accesiones de papa nativa

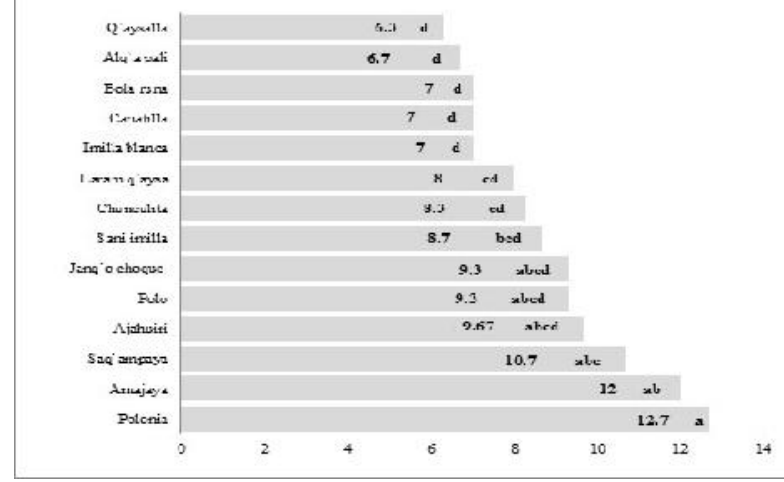

Figura 9 Análisis de comparación de medias mediante la prueba de Duncan $(\alpha=0.05)$ para la variable número de flores para las accesiones de papa nativa

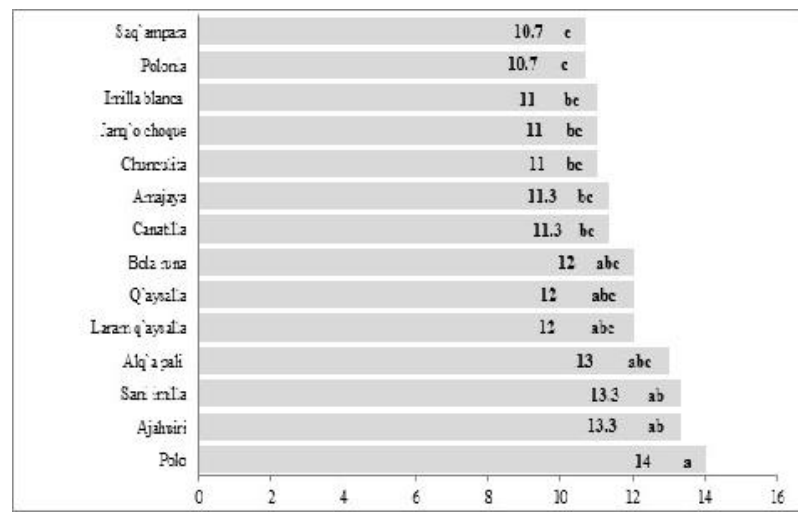


Figura 10 Análisis mediante la prueba de Duncan $(\alpha=0.05)$ para la variable cobertura foliar

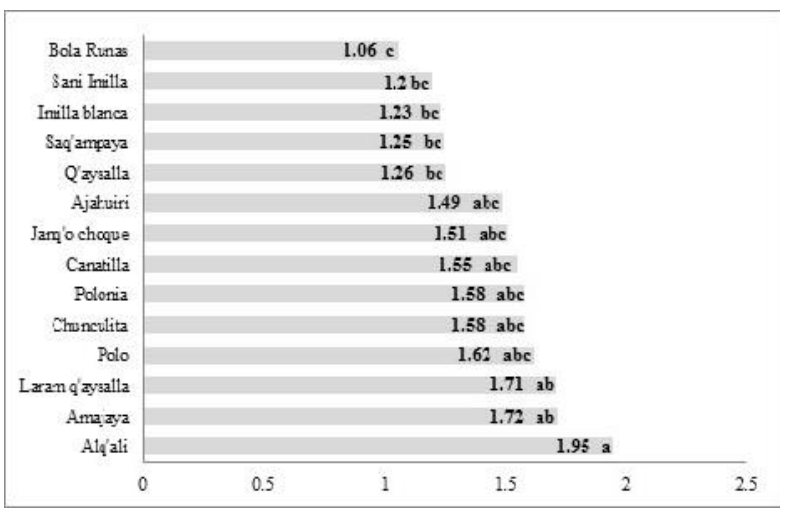

Figura 11 Análisis de comparación de medias mediante la prueba de Duncan $(\alpha=0.05)$ para la variable número de tubérculos de papa nativa (Solanum tuberosum)

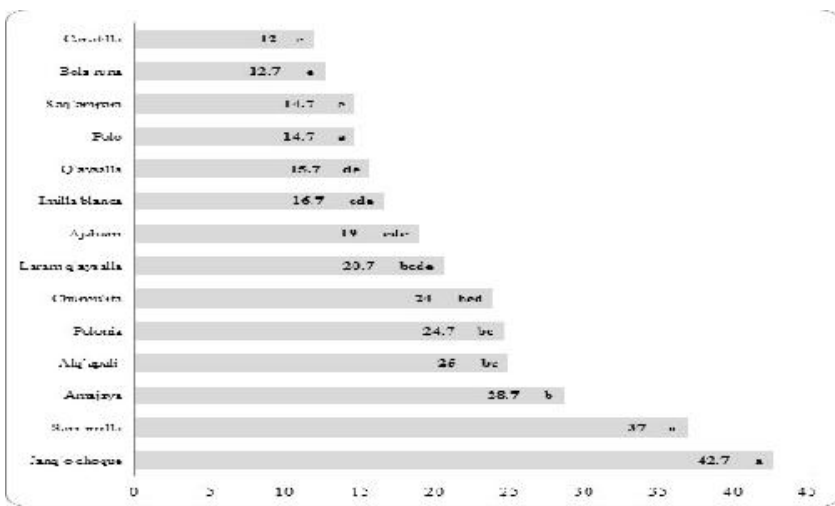

Figura 12 Rendimiento de tubérculos t.ha ${ }^{-1}$

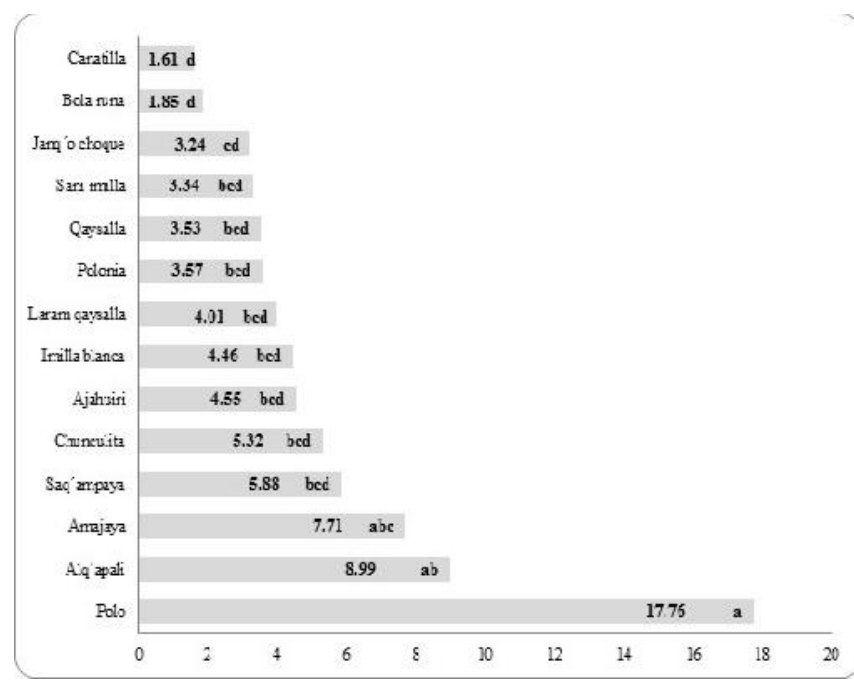

Tabla 4. Análisis de varianza de la eficiencia a los tratamientos en la aplicación de biol en las variables agronómicas

\begin{tabular}{|c|c|c|c|c|c|c|c|}
\hline \multirow[t]{2}{*}{ Fuente variación } & \multirow[t]{2}{*}{ GL } & \multicolumn{2}{|c|}{$\mathbf{C F}$} & \multicolumn{2}{|c|}{ NT } & \multicolumn{2}{|c|}{ REND } \\
\hline & & CM & Pr $>$ F & CM & Pr $>$ F & CM & Pr $>$ F \\
\hline BIOL (Bloq.) & 2 & 4.56 & 0.577 & 3.31 & 0.133 & 39438.373 & 0.139 \\
\hline $\mathrm{ACC}$ & 13 & 16.358 & 0.064 & 3.663 & 0.027 & 59315.591 & 0.006 \\
\hline Error & 26 & 8.111 & & 1.515 & & 18526.197 & \\
\hline Total & 41 & & & & & & \\
\hline $\mathrm{CV}$ & & & 10.41 & & 21.26 & & 11.96 \\
\hline $\mathrm{R}^{2}$ & & & 0.511 & & 0.853 & & 0.638 \\
\hline
\end{tabular}


Tabla 5 Matriz de correlación de 7 variables agronómicas, a factores de aprovechamiento de biofertilizantes en las accesiones de papa nativa

\begin{tabular}{crrrrrrr}
\hline Variables & AP & NR & \multicolumn{1}{c}{ LR } & CF & NF & NT & \multicolumn{1}{c}{ REND } \\
\hline AP & 1.0 & $0.408(* *)$ & $0.872(* *)$ & $0.665(* *)$ & 0.205 & 0.088 & $0.453(* *)$ \\
NR & & 1.0 & $\mathbf{0 . 3 7 2}(*)$ & 0.240 & -0.154 & 0.231 & 0.154 \\
LR & & & 1.0 & $0.732(* *)$ & 0.131 & 0.135 & 0.288 \\
CF & & & & 1.0 & 0.011 & 0.257 & $\mathbf{0 . 3 1 9}(*)$ \\
NF & & & & & 1.0 & -0.090 & 0.190 \\
NT & & & & & 1.0 & 0.169 \\
REND & & & & & \\
Altura de planta AP, número de ramas NR, largo de rama LR, cobertura foliar CF, número de flores NF, \\
número de tubérculos NT, Rendimiento REN
\end{tabular}

Tabla 6 Estadística descriptivas sobre variables agro morfológicas del estudio caracterización de 14 accesiones de papa nativa

\begin{tabular}{|c|c|c|c|c|c|c|c|}
\hline & $\mathbf{A P}$ & NR & $\mathbf{L R}$ & $\mathbf{C F}$ & NF & NT & REND \\
\hline Promedios & 29.090 & 8.762 & 31.981 & 27.348 & 11.905 & 22.000 & 3597.8 \\
\hline Media & 27.635 & 8.000 & 32.165 & 27.830 & 12.000 & 18.500 & 3167.7 \\
\hline Deviación Estand. & 7.3692 & 2.5067 & 6.1007 & 3.2504 & 1.5111 & 9.7079 & 1802.206 \\
\hline Sesgo & 1.076 & 1.045 & 0.479 & -0.003 & 0.080 & 1.281 & 1.346 \\
\hline Curtosis & 2.033 & 0.727 & 0.599 & 0.480 & 0.650 & 1.160 & 1.970 \\
\hline Mínima & 15.3 & 5.0 & 18.1 & 20.2 & 8.0 & 11.0 & 1003 \\
\hline Máxima & 53.5 & 16.0 & 47.7 & 36.0 & 16.0 & 50.0 & 17767.760 \\
\hline
\end{tabular}

\section{Discusión}

De acuerdo a la Figura 4, el nitrógeno es el elemento predominante en la composición elemental del Biol obtenido, $0.34 \%$ en base a materia seca, bajo comparado con los datos obtenidos por (Rodríguez 2003), quien obtuvo valores en torno al $1.48 \%$ mediante la biodigestión de estiércoles de ganado camélido, ovino y bovino en la localidad de Taraco. De todas maneras las concentraciones se sitúan muy por debajo a los reportados como $2.6 \%$ (Jairo et al. 2007) en experimentos realizados con estiércol bovino biodigeridas. Los datos contrastan concentraciones de nitrógeno del orden de 2.5 a $3.5 \%$, similares a (Piedrahita 2000), con valores entre $2.0 \%$ a $2.6 \%$. Es llamativa también la gran diferencia respecto a los datos de (Zagas et al. 2000) que reportaron $\mathrm{N}$ total de $3.7 \%$.

En función a lo mencionado, resulta claro que la composición del Biol depende de la dieta a la que 23 son sometidos los animales, además de factores ambientales, época del año y el manejo que fue sometido el material previamente a su fermentación. La reducción en los niveles de nitrógeno en forma nítrica $\left(\mathrm{NO}_{3}\right)$, en mezclas biodigeridas también fueron advertidas por (Zagas et al. 2000) quien atribuye este efecto a la desnitrificación que ocurre por ausencia de oxígeno en el interior del biodigestor durante la transformación del $\mathrm{N}$ orgánico en formas amoniacales $\mathrm{NH}_{4}$.

En lo que respecta a los niveles de fósforo el valor se estuvo $0.04 \%$, que resulta bajo a los obtenidos por (Piedrahita 2000), que obtuvo $1.4 \%$ en proceso de biodigestión de estiércol de vacunos. Sin embargo (Zagas et al. 2000), menciona valores de 0.9 a $2.1 \%$ de fósforo en forma de $\mathrm{P}_{2} \mathrm{O}_{5}$ en el efluente de un biodigestor tubular de polietileno.

En cuanto al Calcio y de otros elementos como P y $\mathrm{K}$, no existen pérdidas apreciables durante el proceso de fermentación anaeróbico, como (Piedrahita 
2000). En este sentido es posible deducir que las concentraciones en la materia prima utilizada en el presente estudio fueron relativamente inferiores al promedio para excretas bovinas.

En la composición de micronutrientes en el Biol destaca la presencia de hierro (132 ppm), seguido de Manganeso, Cobre y Zinc con 12.01 .0 y 6.0 ppm respectivamente. Los valores de hierro $(\mathrm{Fe})$ son elevados en relacion a los obtenidos por (Piedrahita 2000), que reporto 4 ppm para este elemento. En cuanto a $\mathrm{Mn}, \mathrm{Cu}$ y $\mathrm{Zn}$, valores que se encuentran dentro del rango de 1 a 6 ppm de manera similar a los reportados por estos autores. (Figura 5)

De acuerdo con la variable AP se observa que no existen diferencias significativas en el factor Biol, es decir, el producto aplicado en las diferentes accesiones de papa nativas no tuvo efecto significativo en el desarrollo AP en sus diferentes dosis. Sin embargo, existe significancia $(\mathrm{P}=0.05)$ respecto al factor Accesiones, por lo que se procedió al análisis de comparación entre medias (tabla 3 ).

El análisis de varianza de LR, no se detectó significancia respecto al factor dosis de Biol, sin embargo, existen diferencias al interior del factor de las Accesiones para lo cual se procedió al análisis comparativo entre medias (tabla 3).

Con relacion a NR no muestra diferencias significativas, probablemente fueron influenciadas por el medio ambiente respecto a la aplicación de dosis de Biol. La variable NF que no hubo efecto del Biol sobre la floración de las diferentes accesiones de papa nativa.

Conforme que se observa en la figura 6 , existen tres grupos diferenciados de accesiones respecto a la altura de planta. Se puede apreciar que la variedad "Alq'a pali" (leke Pek'e), "Polo", "Polonia" y "Amajaya" alcanzaron los mayores altura, fluctuando entre 33.8 y $40.4 \mathrm{~cm}$, respectivamente. Por otra parte las variedades de "Canatilla", "Janq'o cho- que", "Imilla blanca", "Ajahuiri”, "Saq'ampara", "Q'aysalla", "Sani imilla" y "Layam q'aysa" alcanzaron valores entre 29.0 y $23.4 \mathrm{~cm}$.

Por su parte "Chunculita" "Bola runa" fueron las accesiones que presentaron con la menor promedio de 23.4 y $21.9 \mathrm{~cm}$, aunque estos valores no son diferentes significativamente de accesiones como "Bola runa" y "Chuculita". Estas diferencias muestran la gran variabilidad genética existente entre las accesiones de papa nativa, conforme descrito por (Mújica 2007), quién menciona que la altura constituye una característica distintiva muy importante para la caracterización genética de papas nativas.

Las diferencias en AP podrían atribuirse a los hábitos de crecimiento conforme a (Cahuana 2002) que reconoce distintos tipos de crecimiento como el Erecto, Semi-erecto, Decumbente, Postrado, Semiarrocetado, tipo herbáceo cuya AP varía de 0.30 a 1 cm de alto, según la accesión, debido a su característica variedad.

Se puede apreciar que las variedades "Alq'a pali" (Leke Pek'e), "Amajaya”, "Polonia” y "Polo" alcanzaron los mayores promedios de largo de rama, fluctuando entre 35.6 a $42.6 \mathrm{~cm}$. Por otra parte, variedades como "Imilla blanca" ("Janq'o choque"), "Ajahuiri”, "Saq'anpaya" o "Canatilla" presentaron promedios intermedios, hasta $28.9 \mathrm{~cm}$ de largo de ramas. A su vez, la accesión "Bola runa" presento la menor LR con $25.9 \mathrm{~cm}$, se procedió al análisis comparativo entre medias (figura 7).

De acuerdo figura 7 se observa desarrollos diferentes en las accesiones de papa nativa, estas características visibles de los largos de ramas son significativas para separar distintos grupos, debido a su característica varietal.

Se puede apreciar que las accesiones "Polonia", "Amajaya”, "Saq'ampaya", "Ajahuiri”, "Polo" y "Janq'o choque" alcanzaron los mayores promedios respecto al número de ramas fluctuando entre $12.7 \mathrm{y}$ 
9.3 unidades respectivamente, valores superiores a los reportados por (Cahuana \& Arcos 1993), que menciona, el número de ramas oscila entre 5 a 10 unidades de ramas por planta (figura 8). Las variedades como "Sani imilla", y "Chunculita" presentaron hasta 8.3 ramas en promedio. La accesión "Q‘aysalla" presentó el menor NR con 6.3 ramas valor que no difirió estadísticamente del número de ramas de las accesiones "Bola runa" o "Alq'apali" (Lekepek’e).

Figura 8, para la variable de NR se pueden evidenciar diferencias entre las accesiones, en particular "Polonia" y "Amajaya" que tuvieron mayor desarrollo debido a sus características varietales.

Al realizar un análisis comparativo de las catorce accesiones de papa nativa (Solanum tuberosum), se observa que el número flores varió entre 10.7 hasta 14 unidades de flores por planta (figura 9). La accesión "Polo" presentó el mayor NF en cuanto que "Polonia" y "Saq'ampaya" presentaron la menor cantidad de flores con un promedio de 10 unidades de flores por planta. De manera similar con la anterior variable, se observan diferencias ente las catorce accesiones.

Tabla 4, el análisis de la variable CF detectó diferencias significativas en el factor dosis de Biol sobre la cobertura foliar de las diferentes accesiones de papa nativa. Este efecto positivo probablemente está relacionado con una dotación óptima de nutrientes y agua con lo cual se logra una cobertura foliar de manera temprana y persistente en los tubérculos andinos. Por otra parte, un efecto positivo sobre el desarrollo foliar puede atribuirse al efecto fitohormonal del Biol, conforme mencionado por (Aparcana-Robles \& Jansen 2008), que enfatiza el efecto promotor y fortalecedor del crecimiento que posee este producto gracias a su concentración de fitohormonas como ácido indolacetico, giberelina, 25 purinas y otras sustancias bioactivas.

Patiño \& Rojas (2000), reconoce la importancia de una mayor cobertura foliar como una variable esencial para la resistencia al estrés, en este sentido la aplicación de Biol puede constituir una opción viable para lograr este cometido, además de nutrir a la planta. En este mismo sentido (Antezana 2001), observa un efecto marcado de la fertilización orgánica sobre el índice de área foliar, duración de follaje, la acumulación de biomasa y cobertura foliar de variedades de papa ("Waycha" y "Pintaboca"). Una mayor cobertura foliar, proporcionada por la aplicación de Biol también puede estar relacionada con un alargamiento en el ciclo de vida de las plantas, con efectos positivos sobre el rendimiento, conforme observado por (García et al. 2003).

El análisis del variable NT no detectó diferencias en el factor de Biol sobre accesiones respecto al variable número de tubérculos. A pesar de ello observa unas leves diferencias entre las distintas accesiones. El análisis de varianza de la variable Rendimiento muestra que no existen diferencias significativas respecto al factor dosis de Biol. Las diferencias entre accesiones muestran la variabilidad genética existente entre ellas con mayores rendimientos para las accesiones.

Figura 10, con respecto a las diferencias entre accesiones se pudo evidenciar que variedades como "Alq‘a pali" ("Lekepek'e"), "Amajaya" y "Laram q'aysalla" presentaron mayores promedios de cobertura foliar que "Sani imilla" y "Bola runa" que presentaron la menor cobertura foliar.

Se puede apreciar que las variedades de "Janq'o choque" y "Sani imilla" alcanzaron los mayores número de tubérculos entre 42.7 y 37 , unidades respectivamente. Por otra parte las variedades de "Amajaya", "Alq'apali", "Polonia", "Chunculita" y "Layam q'aysa" alcanzaron promedios intermedios 
entre 28.0 y 20.7 unidades. "Bola runa" y "Canatilla" produjeron la menor cantidad de tubérculos por planta (entre 12.0 y 14.7 unidades por planta).

Para la variable de número de tubérculos por planta, se encontró que las accesiones Canatilla y bola runa presentaron menor número de tubérculos (12 tubérculos/planta), por otro lado la accesión Saq’ampaya, Polo, Q'aysalla presentaron entre (14.7-16 tubérculos/planta), así mismo también se puede apreciar el mayor número que presentó el promedio para el Janq'o choque donde se observándose que el número de tubérculos por planta fluctúa alrededor de 42 tubérculos. Figura 11.

El rendimiento de tubérculos por planta se situó entre 17.76 y 7.71 t.ha $^{-1}$ lo cual guarda relación con los datos obtenidos por (Quispe 2000), a los 135 días de la siembra. Los datos obtenidos para accesiones como "Polo" condicen con rendimientos obtenidos en papas nativas sometidas a fertilización foliar con Biol, conforme fue constatado por FONTAGRO (2008), en cuyos ensayos se obtuvo rendimientos de 16 t.ha ${ }^{-1}$. Figura 12.

La matriz de correlación simple entre cada variable, cuantitativa tabla 5, del total de coeficientes analizados, siete fueron significativos (marcados con uno o dos asteriscos, según sean significativos o altamente significativos). Se consideró como asociaciones lineales a aquellas que presentan coeficientes superiores a 0.20 .

Por ejemplo, para la variable AP se obtuvo una alta correlación $(0.872 * *)$ con la variable "Largo de ramas", por el contrario no se detectó una relación entre esta variable y NT.

De manera similar, la variable LR presenta una relación significativa con la $\mathrm{CF}$, con un coeficiente $r=0.73$, parámetro que fue influenciado positivamente por el Biol aplicado, conforme fue discutido en el acápite correspondiente. Por otro lado, se observa que no se estableció una correlación significa- tiva entre la CF y NT, sin embargo la relación con el "Rendimiento" alcanzó un $\mathrm{r}=0.319$, tabla 5.

El análisis descriptivo de las accesiones muestra una función de distribución normal (según los coeficientes de sesgo y curtosis). Los resultados obtenidos muestran un rendimiento de 3.59 t.ha $^{-1}$, con un mínimo de 1.0 t.ha ${ }^{-1}$ y un máximo de 17.76 t.ha $^{-1}$ lo cual muestra la gran variabilidad genética entre las accesiones, tabla 6 .

En base a los resultados obtenidos, la aplicación de Biol como fertilizante foliar sobre 14 accesiones de papa nativa no originó un incremento en el rendimiento de tubérculos, pero sin embargo hubo en efecto positivo en el desarrollo de la cobertura foliar.

Se obtuvieron rendimientos elevados para algunas accesiones como "Polo", alcanzando un rendimiento de 17.76 t.ha $^{-1}$ seguido por "Alq'apali" con 8.99 t.ha ${ }^{-1}$. Las accesiones con menores rendimientos bajo las condiciones de estudio fueron "Bola runa" con 1.85 t.ha ${ }^{-1}$ y "Canatilla" 1.61 t.ha ${ }^{-1}$.

Las accesiones "Polonia", "Amajaya", "Saq'ampaya", “Ajahuiri”, "Polo" y "Janq'o choque" alcanzaron los mayores promedios respecto al número de ramas fluctuando entre 9.3 y $12.7 \mathrm{~cm}$. La accesión " $\mathrm{Q}$ ‘aysalla" presentó el menor número de ramas con 6.3 unidades. Con respecto al "Largo de Ramas" la accesión Amajaya" alcanzó 63.9 cm y el menor desarrollo presento "Bola Runa" con $25.9 \mathrm{~cm}$.

\section{Conflictos de intereses}

Los autores han cumplido las normas éticas de publicación, y no generan conflicto de interés en la presente investigación. 


\section{Agradecimientos}

Se agradece al responsable de la Estación Experimental de Kallutaca, dependiente de la Universidad Pública y Autónoma de El Alto (UPEA).

Al Director de Instituto de Investigación y Extensión Agrícola (IINEA). Área de Ciencias Agrícolas, Pecuarias y Recursos Naturales, Ingeniería Agronómica.

\section{Literatura citada}

Antezana LF. Determinación del rendimiento potencial de cultivares priorizados de papa (Solanum tuberosum), oca (Oxalis tuberosa) e isaño (Tropaeolum tuberosum), Toralapa y Candelaria. Departamento-Cochabamba. [Tesis de Licenciatura]. Universidad Mayor de San Simón. Cochabamba-Bolivia. 2001. p. 117.

Aparcana-Robles S, Jansen A. Estudio sobre el valor Fertilizante de los productos del proceso fermentación anaeróbica para producción de biogás, German Prof EC GmbH, Lima, Perú, 2008.

Avilés M, Borrero C, Trillas MI. Compost supresivos a las enfermedades de las plantas. Revista Ae 2011; (3): 6-8.

Bot A, Benites J. The importance of soil organic matter. FAO Soils Bulletin 80. FAO, Rome. 2005. p. 78.

Cahuana R, Arcos J. Variedades de papa más importantes en Puno y lineamientos para su caracterización Piwa (programa Interinstitucional de Waru waru convenio: PELT/ INADE - IC/ COTESU Puno Perú $1^{a}$ Ed. Bolivia. 1993. p 28.
Cahuana R. Gestión del espacio agrícola en las comunidades campesinas de Puno. En: Sepia IX. Perú: el problema agrícola en debate. Puno, Perú. $1^{a}$ ed. Puno, Perú, CIMA. 2002. p. 210.

Estrada N. La Biodiversidad en el Mejoramiento Genético de la papa. Bill Hardy, Emma Martinez (Ed.) La Paz, Bolivia. 2000. p. 372.

Fisher DS. Burns JC, Pond KR. 1989. Kinetics of in vitro cell-wall disappearance and in vivo digestion. Agron J 1989; 18:25-33.

FONTAGRO. En Centroamérica y países Andinos FONTAGRO financia Proyectos sobre papa “Agro-noticias" año 4. 20008. p. 53.

Gabriel J, Pereira R, Gandarillas A. Catálogo de nuevas variedades de papa en Bolivia. Fundación PROINPA, Cochabamba, Bolivia. 2011. p. 55.

Gabriel J. Documento marco: Estrategias y perspectivas del mejoramiento genético de papa (Solanum tuberosum L.) en Bolivia. Fundación PROINPA, Cochabamba, Bolivia. 2010. p. 60.

Gajalakshmi S, Abbasi SA. Eathworms and vermicomposting. Indian J Biotechnol 2004; 3: 48694.

García W, Patiño F, Almanza J, Gonzáles R. Carpeta socioeconómica del Municipio de Colomi. [Tesis de Licenciatura]. Universidad Mayor de San Simón. Cochabamba, Bolivia. 2003. p. 128.

Huamán Z, Spooner DM. Reclassification of landrace populations of cultivated potato (Solanum sect. Petota). Amer J Bot 2002; 89:947-65.

Jairo A, Ciro H, Sánchez H. Evaluación De Un Sistema De Biodigestión En Serie Para Clima Frío. Rev Fac Nal Agr Medellín 2007; 60: 4145-62.

Mujica A. Potencialidades productivas y económicas de la diversidad de cultivos andinos ali- 
menticios en las regiones montañosas. En: Resúmenes II Foro Latinoamericano de Montanas. Universidad Nacional de Jujuy, Foro Permanente de Montanas 11- 12 de septiembre. Jujuy, Argentina. 2007. p. 12.

Ochoa CM. Las papas de Sudamérica: Perú (parte I). 1999. p. 1036.

Ochoa CM. The potatoes of South America: Bolivia. Cambridge University Press, Cambridge, UK. 1990. p. 512.

Ortuño N, Navia O, Medrano A, Rojas K, Torrico L. Desarrollo de Bioinsumos: Un Aporte Importante a la Soberanía Alimentaria de Bolivia. Revista de Agricultura 2010; (47): 31-5.

Patiño J, Rojas F. Rendimiento Potencial de papa nativas (Solanum tuberosum ssp. Andigena y stenotonum), papalisa (Ullucus tuberosus), oca (Oxalis tuberosa) e isaño (Tropaeolum tuberosum), en la localidad de Candelaria. Prov. Chapare-Cochabamba. [Tesis de Licenciatura]. Universidad Mayor de San Simón, Cochabamba-Bolivia. 2000. p. 94.

Piedrahita D. Elementos para una tecnología sobre producción de biogás. Universidad Nacional de Colombia, Medellín. 2000. p. 137.

Quispe M. Caracterización preliminar del banco germoplasma de papas nativas del altiplano norte en la estación experimental de Belén. [Tesis de Licenciatura]. Universidad Mayor de San Andrés. Facultad de Agronomía. La PazBolivia. 2000. p. 123.

Rodríguez W. Datos climáticos. Informe: resumen del período 1996 a 2003 (documento electrónico). Estación Meteorológica, Universidad EARTH. Guácimo, CR. 2003.
Rojas-Rodriguez K, Ortuño N. Evaluación de micorrizas arbusculares en interacción con abonos orgánicos como coadyuvantes del crecimiento en la producción hortícola del Valle Alto de Cochabamba, Bolivia. RevActaNova 2007; 3(4): 697-718.

Thiele G, Hareau G, Suarez V, Chujoy E, Bonierbale M, Maldonado L. Varietal change in potatoes in developing countries and the contribution of the International Potato Center (IPC): 1972-2007. ICP, Lima, Perú. Working Paper 2008-6. 2008. p. 46.

Warren SL, Fonteno WC. Changes in physical and chemical properties of a loamy sand soil when amended with composted poultry litter. J Environ Hort 1993; 11:186-90.

Zagas Th, Ganatsas P, Tsitsoni Th, Hatzistathis, A. Influence of sewage sludge application on survival and early growth of forest species. Proc. of 5th International Conference «Protection and Restoration of the Environment». July 3-6, 2000, Thasos Island. Greece. 2000. Vol. I: 583-90.

Zeballos H, Balderrama F, Condori B, Blajos J. Economía de la papa en Bolivia (1998-2007). Fundación PROINPA, Cochabamba, Bolivia. 2009. p. 129. 